\title{
Natural variation reveals that OsFLA2 controls flag leaf angle in rice (Oryza sativa L.)
}

\author{
Xiaojing Dang ${ }^{1}$, Changmin $\mathrm{Hu}^{1}$, Lei Xu ${ }^{1}$, Ying Zhang ${ }^{1}$, Dezheng Wang ${ }^{1}$, and Jianhua \\ Jiang $^{1}$
}

${ }^{1}$ Anhui Academy of Agricultural Sciences

November 24, 2020

\begin{abstract}
Flag leaf angle (FLA) is an important outcrossing trait affecting the hybrid seed production in rice (Oryza sativa L.). Natural variation of FLA has been reported in rice, but the molecular basis for this variation is largely unknown. Here we investigated the phenotypic values of FLA in 353 rice natural accessions in six environments, which indicated rich phenotypic variation. We performed a genome-wide association study on FLA using 1.3 million single nucleotide polymorphism (SNPs). A total of 37 SNPs were identified significantly associated with FLA, of which 27 were located in previously reported QTLs/Genes and 10 were novel. We identified two causal gene loci for FLA, OsFLA6 and OsFLA2; OsFLA6 was co-localized with the gene OsLIC. In addition, the accessions with large and small FLA values have corresponding high and low OsFLA6 expression. We also confirmed that the allele OsFLA2TT increased the FLA compared with that of the isogenic line carrying allele OsSYL2CC by transgenic complementation experiment. The allele frequencies of OsFLA6GG and OsFLA2TT decreased gradually with an increase in latitude in the Northern Hemisphere. Our results should facilitate the improvement of FLA of parents of hybrid rice.
\end{abstract}

\section{Hosted file}

2020-11-24-Manuscript of FLA for PCE.pdf available at https://authorea.com/users/378452/ articles/494939-natural-variation-reveals-that-osfla2-controls-flag-leaf-angle-in-riceoryza-sativa-1

\section{Hosted file}

2020-11-24-Figures.pdf available at https://authorea.com/users/378452/articles/494939natural-variation-reveals-that-osfla2-controls-flag-leaf-angle-in-rice-oryza-sativa-l 